\title{
Double Circuit EHV Transmission Lines Fault Location with RBF Based Support Vector Machine and Reconstructed Input Scaled Conjugate Gradient Based Neural Network
}

\author{
K. Gayathri * \\ Department of Electrical Engineering, Annamalai University, \\ Annamalainagar-608 002, Tamilnadu, India. \\ e-mail:g.gayathri3@gmail.com \\ N. Kumarappan \\ Department of Electrical Engineering, Annamalai University, \\ Annamalainagar-608 002, Tamilnadu, India. \\ e-mail:kumarappan_n@yahoo.com
}

Received 10 November 2013

Accepted 19 June 2014

\begin{abstract}
A new algorithm is developed to enhance the solution for the problems associated with double circuit transmission lines for the mutual coupling between the two circuits under fault conditions and which is highly variable in nature. The algorithm depends on the three-line voltages and the six line currents of double circuit lines at one end. It relies on the application of Support Vector Machine (SVM) and frequency characteristics of the measured single end positive sequence voltage and current measurement of transient signals of the system. Fault resistance, mutual coupling between two circuits and initial prefault conditions are considered. The accuracy of this method has been assessed using a fault simulation software program. In the first state, the accuracy of the method was determined on the basis of SVM reconstructed method. In the second state, this method utilizes voltage and current data acquired at one common end of the two lines. This paper proposes a new hybrid approach for fault location on Extra High Voltage (EHV) lines using RBF based SVM with reconstructed input and Scaled Conjugate Gradient (SCALCG) based neural network method. Sample inputs are determined by MATLAB. The average error fault location in $400 \mathrm{kV}$ and $150 \mathrm{~km}$ line is tested and the results prove that the proposed method is effective and reduces the error within a short duration of time using both RBF based reconstructed input of SVM and SCALCG based neural network.
\end{abstract}

Keywords: Double Circuit; EHV transmission line; Fault locator; Reconstruction; Radial basis function; Support vector machines; Neural network .

${ }^{*}$ Corresponding author. 


\section{Introduction}

The parallel transmission lines are considered the most vital components in power systems for occurrence of line faults; the maintenance crew must find the problem to restore the service as quickly as possible. The rapid restoration of the service reduces outage time and loss of revenue. Therefore, the accurate fault location under a variety of fault conditions is an important requirement. So far, several techniques have been adopted for pattern recognition of the high frequency signals generated. However, double circuit lines in general are more difficult to protect using conventional distance protection schemes because of the mutual coupling between the two circuits and the possibility of inter circuit faults occurring on such a system. Therefore, it is essential to develop an alternative fault classifier to analyze the fault on the double circuit transmission line.

One of the most important task for the protection of power transmission lines is to classify the type of fault if that a single-phase-to-ground, double phase-to-ground, phase-to-phase, or a three-phase type. Once the type of fault is classified, it is then important to find the location of the fault. In this paper the fault location method applicable to double circuit transmission lines, is based on the determination of a particular distance factor for a given fault. The definition of a new concept called the 'distance factor' will allow a method to be developed which is independent to fault resistance, type of fault, mutual coupling between the two circuits and initial pre fault conditions [1]. The problems encountered by conventional distance relays while protecting double-circuit transmission lines comprising different voltage level are addressed in [2]. An algorithm for the computation of fault location in transmission lines by means of the voltage and current signals, acquired by the digital distance relays at both the terminals of the lines is presented in [3]. A fault location method for one or two individually transposed parallel transmission lines has been developed. This method utilizes only the voltage and current data obtained at one common end of the lines to estimate the equivalent source impedance at the remote end [4]. Fault location in two terminal overhead transmission lines, using ANN is based on fundamental components of the fault and pre-fault voltage and current magnitude measured in each phase at the reference end are discussed in [5]. The potential applicability of ANN techniques for determination of fault location and fault resistance on EHV transmission lines with remote end in feed is presented in [6]. An algorithm for estimating fault location on a transmission line is studied and tested using simulation data obtained from the Electro Magnetic Transient Program (EMTP) [7]. A particle fault location approach for double circuit lines is introduced and data is extracted from one end of the line [8]. The design and implementation of an artificial neural networks based fault locator for EHV transmission lines are presented in [9]. The location of fault has been presented in the high-voltage power transmission line, support vector machine and frequency characteristics of the measured one-terminal voltage and current transient signals of the systems are reported in [10]. The fault location algorithm for the parallel lines with measurements at one end is delivered in [11].

Neural space vector to fault location for parallel double circuit distribution power lines is presented in [12]. An ANN based application for fault location in electrical transmission lines has been presented in [13]. The fault location has been investigated on parallel transmission lines using wavelets for accurate rapid fault detection and fault location estimation regardless of mutual coupling between parallel lines are considered in [14]. Pre-processing the fault current signal samples using discrete wavelet transform to yield the change in energy (ce) and standard deviation (sd) at the appropriate level of decomposition of fault current and voltage signal for fault phase identification and fault location determination are developed [15]. Fault location method to locate a fault has been presented in a single multi-terminal transmission line [16]. Fault location in transmission lines are presented, using the calculation of fault distance phasor at voltage and current at one of the line terminals, and also the system equivalents at both line terminals [17]. Fault location formulas using synchronized sequence voltage and current at two-ends of faulted transmission lines are presented in [18]. Artificial neural networks [19] are used to classify and locate the faults and wavelet transform [20] is also used to identify and classify the faults. But the drawback of this method is the inefficiency in the resolution.

In this paper, efficient resolution, sequence voltage and current, frequency are used and features from both fault voltage and current signals are used to train and test the Radial Basis Function Neural Network 
(RBFNN). For simulation and testing of the proposed approach, faults are created at a double circuit line fed from both ends with variations in source impedance and fault resistance. The proposed approach is used to locate double line to ground faults for double circuit with wide range of operating conditions. The important feature of SVM is, it aims at limited samples, increased complexity with modest computational effort and high matrix is solved accurately and effectively. It always finds a global solution for optimization model, good theory and practical expression of tradeoff between learning and generalization. The fault location algorithm for the parallel lines delivered is very simple and compact.

\section{Simulation Studies for EHV Double Circuit Transmission Line}

The double circuit transmission line is significantly used to increase the transmission capacity on existing systems and is finding more wide spread usage. It is also advantageous than single circuit. When conventional technique is applied to parallel lines, the performance is affected by the mutual coupling between the lines. The mutual inductance between pairs of conductors in the two lines is not the same and therefore, emf is produced in the conductors of the other line. Fig.1 shows the system under study and Fig.2 shows simulation model of EHV double circuit transmission line. This circuit consists of twotransmission lines consisting of generator at both ends with $150 \mathrm{kms}$ of length with $400 \mathrm{kV}$. The two lines shown are one pair of parallel transmission lines which run in the same right of way, so herewith mutual lines are considered. The transmission line is represented by Pi sections (3 Pi sections are used for each line) and its parameters frequency dependence is taken into consideration for mutual coupling.

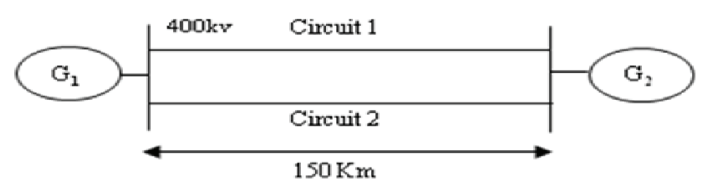

Fig.1. Power system under study

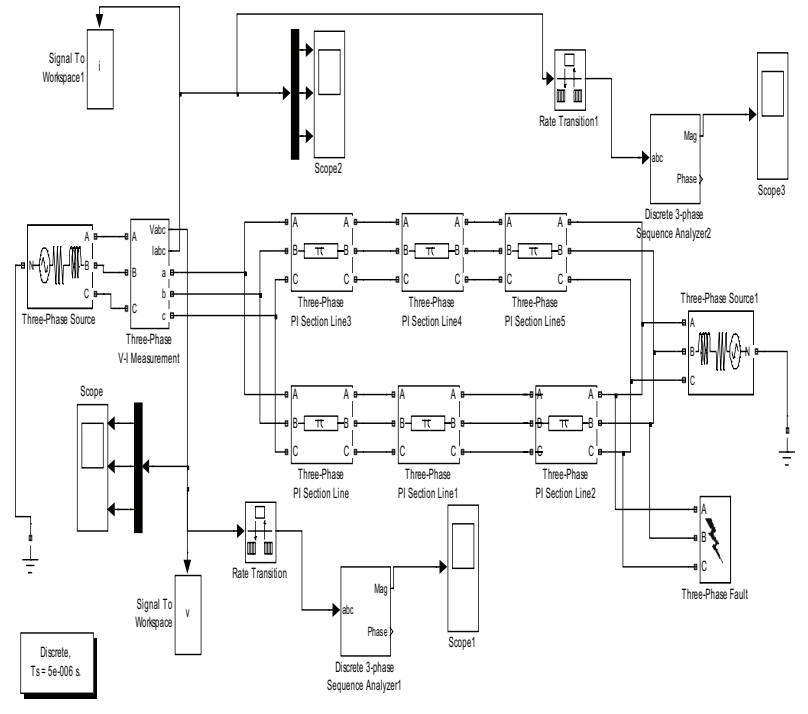

Fig.2.Simulation model of EHV double circuit transmission line

Fig.3 shows the implementation diagram of SVM method. The voltage and current waveforms of the simulated power system are fed as input to the sampling network. The signals are sampled at $5 \mathrm{kHz}$ to obtain higher resolution. The sampled signals are given to the SVM to locate the faults. Thus faults are located for the EHV transmission line with mutual coupling.

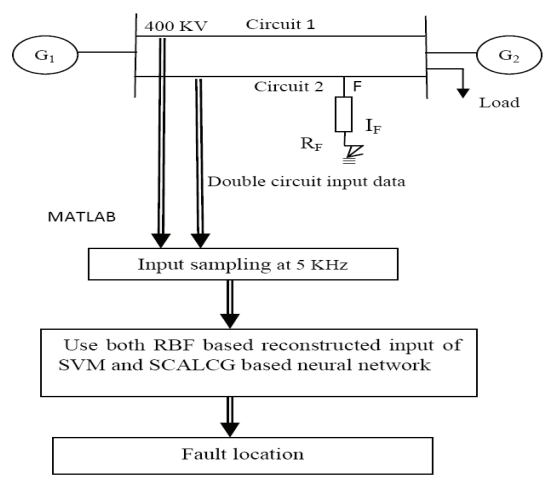

Fig. 3. Implementation diagram of SVM method

The methodology of SVM offers an elegant and efficient technique for obtaining or learning general nonlinear discriminate functions using a given set of training patterns in a pattern recognition problem. The description of RBF based reconstructed input of SVM location, frequency characteristics based SVM location 
and finally SCALCG based neural network location regressions are presented in the following sections.

\section{RBF Based SVM Method for Fault Location on EHV Line}

\subsection{Input analysis}

The positive sequence voltage, positive sequence current waveforms and frequency characteristics of the simulated power system using MATLAB packages are used to obtain the features of the faulted system. These signals are to be fed to the sampling network where waveforms are sampled at $5 \mathrm{kHz}$. Necessitate for sampling is to view the fault features in elaborate manner.

\subsection{SVM methodology}

To solve the problem of fault location in the power transmission line the SVM approach has been applied. The most important problem in solving any task using neural network is generation of the features and characterizing the process in the best unique way. Then algorithm of SVM is as follows

1. Obtain V, I, max harmonics and fault distance from simulink power system block

2. Scale the value of V, I, max harmonic and distance of fault $[+1,-1]$ or $[1,0]$

3. Select a suitable kernel.

i. Linear $K\left(x_{k}, y_{k}\right)=x_{k}^{T} y_{k}$

ii. Polynomial $K\left(x_{k}, y_{k}\right)=\left(\gamma x_{k}^{T} y_{k}+r\right)^{d}, \gamma>0$.

iii. $\mathrm{RBF}$

$$
K\left(x_{k}, y_{k}\right)=e^{\left(-\gamma\left\|x_{k}-y_{k}\right\|^{2}\right)}, \gamma>\mathbf{O}
$$

iv. Sigmoid $K\left(x_{k}, y_{k}\right)=\tanh \left(\gamma x_{k}^{T} y_{k}+r\right)$

In "Eq.(1),(2),(3),(4)" where, $\mathrm{K}$ is the linear dot product or a nonlinear function obeying Mercer's condition, $x_{k}$ is the input training vectors, $y_{k}$ is the support vectors, $\mathrm{d}$ is the destination, $\mathrm{r}$ and $\gamma$ are the kernel parameters. (From the above kernels, RBF kernel is proposed in this paper).

4. Find $\mathrm{C}$ and $\gamma$ parameter of kernels, where $\mathrm{C}$ is the user specified parameter.

5. Perform coarse grid search

6. Further perform a finer grid search on that region. After this repeat step 4 for all training sets
7. The final classifier gives the trained SVM for power system fault location

\subsubsection{Reconstruction method}

Steps for input space reconstruction method:

i) Use RBF kernel is used for extraction of the principle eigenvectors of the feature space from the given inputs.

ii) Make a grid over the input space.

iii) Project each point of the input space with the principal eigenvectors

iv) Reduce the error between the principal eigenvector and input space using the denoising function.

This is called reconstruction error minimizing. This can be carried out on the given feature space for SVM classification. Denoising can also be done using RBF kernel [9].

\subsubsection{Flow chart}

The flow chart of the proposed method is clearly shown in Fig. 4.

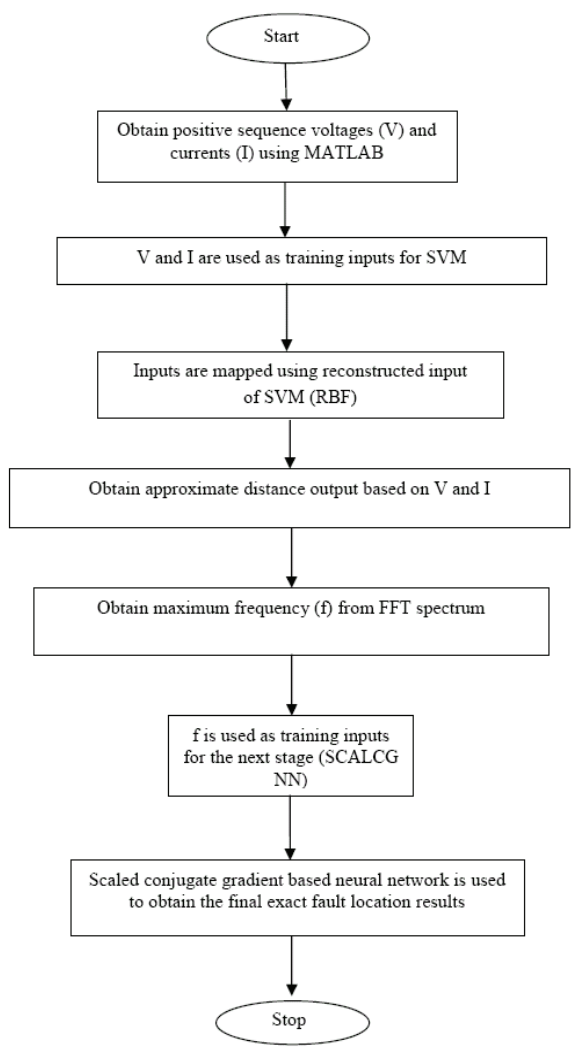

Fig. 4. Proposed method's flow chart 
The description of $\mathrm{V}$ and I inputs with RBF based SVM along with reconstructed input, frequency characteristics based SVM to locate the distance and further to locate the distance accurately SCALCG based $\mathrm{NN}$ is presented in the following sections.

\subsubsection{Radial basis function}

Linear SVM is trained with both separable data and non separable data. Normally the analysis for general casenonlinear machines trained on non-separable data gives the results in a very similar quadratic programming problem. For separable data, when applied to nonseparable data there is no feasible solution and this is evidenced by the objective function growing arbitrarily large. Hence, linear function is not appropriately suited for this proposed work. Scaling is very important for both linear and polynomial kernel, it makes large attributes [training values inputs] which may have computational difficulties. From the above kernels, RBF kernel is selected for the proposed approach. The performance of the RBF network is trained using scaled conjugate gradient approach which selects the location of the faults from the training patterns. The radial basis function has a maximum of 1 when its input is 0 . Hence, in the proposed work RBF kernel as been chosen for locating a fault and the algorithm is presented in [22]. In the first stage, Radial basis function algorithm is used then from broader region identify intensive region.

\section{SCALCG Based Neural Network Method for Fault Location on EHV Line}

\subsection{Scaled conjugate gradient algorithm}

In second stage Scaled conjugate gradient (SCALCG) based neural network is used for getting finer and better location of fault. Having in view the above developments and the definitions of the gradient $g_{k}$, combination of input training vectors $s_{k}$ and $y_{k}$ is the label that represents the security index, as well as the selection procedures for scaling factor $\theta_{\mathrm{k}}+1$, scaled conjugate gradient algorithms can be presented $[21,22]$.

\subsection{Generation features of the hybrid method}

In the hybrid approach, the values of the magnitudes of the fundamental harmonics of the positive sequence voltages and currents of the faulty phases and also the frequency characteristics of the measured voltage in high frequency range are considered to locate the fault. RBF based SVM and SCALCG based NN approach is applied to solve the problem of FL in the power transmission line. The most important problem in solving any task using NN is generation of the features and characterizing the process in the best unique way. The solution presented here relies on two kinds of information. One is the positive sequence voltages and currents at one terminal of the system and the other is the frequency characteristics in high-frequency range of the measured transient voltage at the same terminal of the transmission line.

\section{(i) Positive sequence voltages and currents based SVMFL}

In this approach the three phase transient positive sequence voltages and currents of the $150 \mathrm{~km}, 400 \mathrm{kV}$ transposed transmission line under double line to ground fault condition is considered. The location of fault on fundamental harmonics of voltages and currents will not produce very accurate results, since, in practice, the value of the short circuit resistance is generally unknown. The frequency characteristics are also considered for better estimation of the distance to the fault of the faulty system in high frequency range, which is usually neglected in literature.

\section{(ii) Frequency based SVM FL}

The first step in the analysis of frequency characteristics is to uncover higher harmonics by high- pass filtering of the measured voltage or current transients. The best result is achieved after numerical experiments performed for different shorting resistances and at different moments of fault occurrence. The cut-off frequency and length of the measuring window depends on the type of the fault under investigation within the range of $5 \mathrm{kHz}$. Filtering the positive sequence voltage and current signals uncovers the high frequency range. The frequency characteristics of discrete transform of voltage using SVM is shown in Fig.5(b) There is a visible peak corresponding to the frequency $\mathrm{f}_{\text {max. }}$ of 1350 $\mathrm{Hz}$. This frequency is found to be the function of the fault distance.

Moreover, the frequency $\mathrm{f}_{\max }$ of $1350 \mathrm{~Hz}$ is highly insensitive to the value of short circuit resistance. The angle of the source voltage signal at different faulty conditions has confirmed this shape of frequency characteristics in the high frequency range at the double line to ground fault. However, the range of the fault uses this relationship to give better and accurate FL. This is 
explained in the following section and is used in the hybrid SVM approach of the FL.

Generally in electromagnetic transient simulations the most familiar method is to use Pi sections model. A simple $\mathrm{Pi}$ section model will give the correct fundamental impedance. Pi section is suitable for very short lines where the travelling wave models cannot be used. The validity of Pi section model is restricted to relatively short lines or cables and in general, their frequency response is only good in the neighborhood of the frequency at which the parameters are evaluated. The proposed approach validity is proved to be effective and accurate in Pi section models. Pi section models find the accurate type of fault and location of fault, because it is suitable for short lines, if it is a long line it can be divided into equal parts (proposed power system under study is with the length of $150 \mathrm{~km}$. It is divided into three parts, each part has $50 \mathrm{~km}$ length) there by finding the accurate location of the fault.

\subsection{EHV transmission line fault location by hybrid approach}

In the first stage SVM with reconstructed RBF inputs related to the fundamental component of positive sequence voltage and current of all faulty phases determines the initial placement of the fault. The second stage uses the high-frequency range characteristics of the power system in the vicinity of the initially located fault and measured peak frequency of the actual system for precise estimation of the distance to the fault. The two succeeding stages of hybrid approaches applied on double line to ground fault are

1. Apply the SVM neural network in the first stage, the RBF based SVM with reconstruct input neural network are formed by the amplitudes of the first harmonics of voltages and currents of ill phases of the transmission line. The number of hidden radial units is adjusted in the learning procedure and is equal to the number of support vectors. The SVM network has only one output, whose signal indicates the initial placement of the fault.

2. In the second stage, the measured peak frequency value is compared with the high frequency characteristics of the power system in the neighborhood of the place indicated by SVM. This comparison corrects the initial placement to the final estimation pointed by the actually measured frequency $f_{\max }$. To get the best results of location, the high frequency range characteristics is used in all cases corresponding to all applied short circuit resistance values that is used for learning the SVM network.

Once the network has been trained, it can be used in the retrieval mode, in which the input variables to SVM are determined, as well as the value of $f_{\max }$. The trained hybrid system, corresponding to the appropriate type of fault identifies the exact location of the fault in the line.

\section{Result and Discussion}

The data used in learning have been generated using MATLAB and then preprocessed according to the procedure is described. In learning the chosen data corresponding to the fault is occurred each and every place of the $150 \mathrm{~km}$ transmission line. The short distance $(50 \mathrm{~km})$ discrimination has been assumed to increase the information on the line operating conditions, very essential for obtaining good generalization properties of neural network. It has been observed that the neural network fulfills the role of universal approximation and that more learning data means its better approximation ability. The proposed work is also applicable for the utility system.

If a fault occurs the voltage will drop, which is shown in the SVM input voltage waveforms for double line to ground (a-b-g) fault. The method uses the so called hybrid approach, composed of two steps. In first one, the SVM estimates the initial distance to the phase of fault using the information contained in the positive sequence voltages and currents of faulty phases. The second step improves the final estimation of this distance by using the information contained in the high frequency range characteristics. From this positive sequence voltages and currents are selected and then reconstructed input is given to the SVM fault locator using RBF and SCALCG method.

The resulting decision boundary is plotted in Fig. 5(a). In that data the circle stands for points with label $\mathrm{Yi}=+1$, crosses stand for points with label $\mathrm{Yi}=-1$ with $\mathrm{x}$ axis denoting first phase positive sequence voltage in $\mathrm{kV}$ and $\mathrm{y}$ axis denoting second phase positive sequence voltage in V. From this the data is plotted in Fig. 5(a), where squares stand for points with label $\mathrm{Yi}=+1$, crosses stand for points with label $\mathrm{Yi}=-1$ and data points are indicated by the numbers, this is used to identify each data points and just locate the input data points. The contour plotted in green separates class +1 


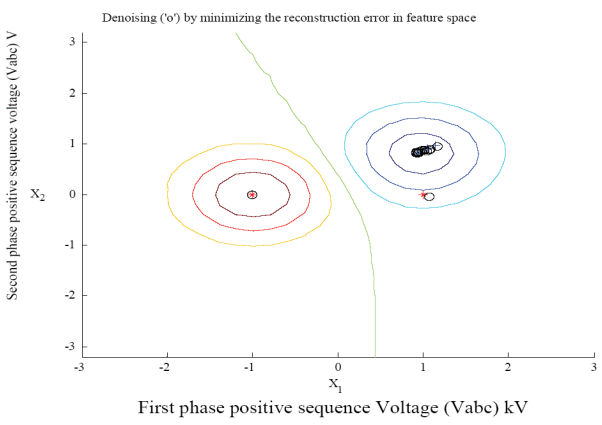

Fig. 5(a). Decision boundary plot of RBF based SVM with reconstructed input for double circuit of double line to ground fault

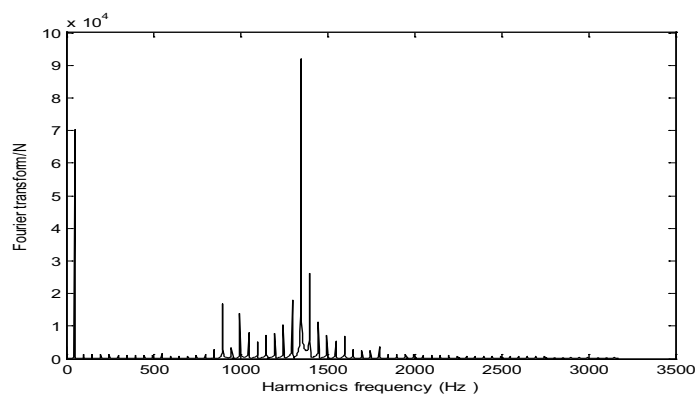

Fig. 5(b). Results of frequency characteristics of the filtered signal in the high frequency range

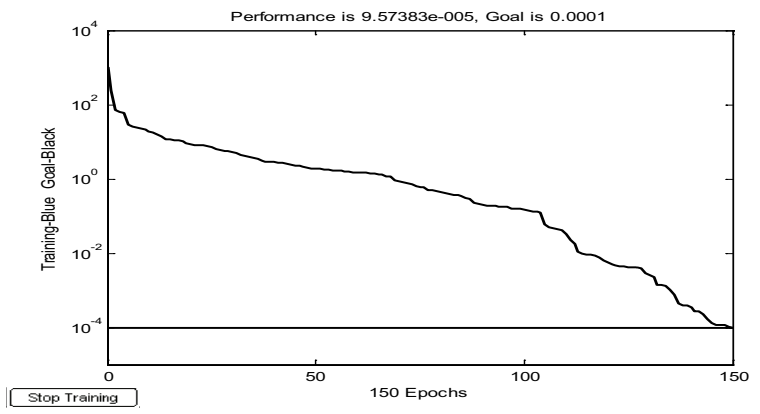

Fig. 5(c)(i). Fault location training performance of Hybrid approach for double line to ground fault $(0-50 \mathrm{Km})$

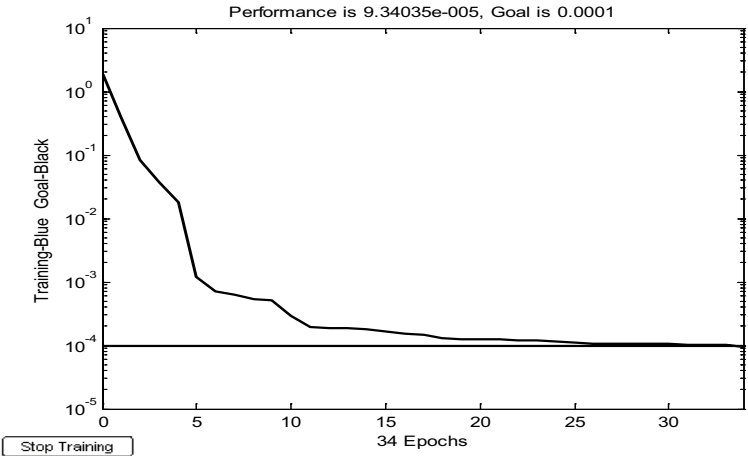

Fig. 5(d)(i). Fault location training performance of Hybrid approach for double line to ground fault $(50-100 \mathrm{Km})$

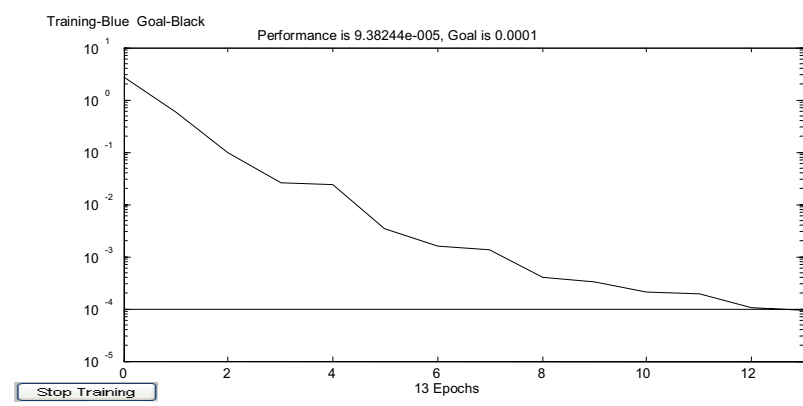

Fig. 5(e)(i). Fault location training performance of Hybrid approach for double line to ground fault (100-150Km)

from class -1 (This is the actual decision boundary). The contour plotted in blue are the points at distance +1 from the decision boundary, the orange contour are the points at distance -1. All examples plotted in red are found to be support vectors. SVs are the examples at distance +1 or -1 from the decision boundary and all the examples that cannot be classified correctly. The data set shown can be correctly classified using a RBF kernel. The upper bound $\mathrm{C}$ for the coefficients has been set and if any of the coefficients are at the bound; this means that all examples in the training set can be correctly classified by the SVM. All the points (support vectors) found to be in the boundary region, in which fault location have been found among the points and used to locate the fault accurately by SCALCG NN method.

Fig. 5(b) shows the results of frequency characteristics of the discrete transform of voltage using SVM with the values of frequency equal to $1350 \mathrm{~Hz}$. N = length (y_fft), where $\mathrm{y}_{-} \mathrm{fft}=\mathrm{y}$ sample; $\mathrm{y}$ is the name of the signal for fft array $\mathrm{Va}, \mathrm{Vb}, \mathrm{Vc}$, or $\mathrm{Ia}, \mathrm{Ib}, \mathrm{Ic}$. This is used in the hybrid SVM approach of the FL. Finally the result shown in Fig. 5(c)(i) is the result of FL training performance of hybrid approach of double circuit with $\mathrm{R}_{\mathrm{f}}=40 \Omega, \mathrm{R}_{\mathrm{g}}=10 \Omega$ for double line to ground fault $(0-50 \mathrm{Km})$. Fig. $5(\mathrm{c})$ (ii) is the FL error of hybrid approach of double circuit with $\mathrm{R}_{\mathrm{f}}=40 \Omega, \mathrm{R}_{\mathrm{g}}=$ $10 \Omega$ for double line to ground fault of the distance $(0$ $50 \mathrm{~km})$. The above results are the combination of both RBF based reconstructed input of SVM and SCALCG based neural method. Similarly the other results are shown in Fig. 5(d)(i), 5(d)(ii) for the distance 50-100km and Fig. 5(e)(i), 5(e)(ii) for the distance 100-150km.

Fig. 5(c) (i) shows the performance of fault locator using hybrid approach of double circuit for double line to ground fault $(0-50 \mathrm{~km})$ with 150 epochs on $\mathrm{x}$-axis and 
the training performance is $9.57383 \mathrm{e}^{-005}$ in blue and goal is 0.0001 in black on y-axis with $\mathrm{R}_{\mathrm{f}}=40 \Omega, \mathrm{R}_{\mathrm{g}}=10 \Omega$. Similarly Fig. 5(d) (i) shows the performance of fault locator using hybrid approach of double circuit for double line to ground fault (50 to $100 \mathrm{~km}$ ) with 34 epochs on $\mathrm{x}$-axis and the training performance is $9.34035 \mathrm{e}^{-005}$ in blue and goal is 0.0001 in black on $\mathrm{y}$ axis with $\mathrm{R}_{\mathrm{f}}=40 \Omega, \mathrm{R}_{\mathrm{g}}=10 \Omega$. Similarly Fig. 5(e) (i) shows the performance of fault locator using hybrid approach of double circuit for double line to ground fault $(100-150 \mathrm{~km})$ with 13 epochs on $\mathrm{x}$-axis and the training performance is $9.38244 \mathrm{e}^{-005}$ in blue and goal is 0.0001 in black on $y$-axis with $\mathrm{R}_{\mathrm{f}}=40 \Omega, \mathrm{R}_{\mathrm{g}}=10 \Omega$, with $400 \mathrm{kV}, 150 \mathrm{~km}$ EHV transmission lines data with learning rate $\eta=0.05$, sampling time $T s=2 \mathrm{e}^{-004} \mathrm{sec}$. The search process is suitable for all type of faults with different training options. The results are obtained by considering double line to ground fault.

The results reveal that the SVM (RBF) with NN (SCALCG) is able to find the fault accurately. It is also possible to locate fault distance for all other type of faults. The proposed approach is fast because the time duration is $2 \mathrm{e}^{-004} \mathrm{sec}$, within this time duration it locates the distance of the fault after the fault occurrence. Hence this proposed approach is better because the fault is located within a time duration of $2 \mathrm{e}^{-004} \mathrm{sec}$ and it is also accurate and efficient.

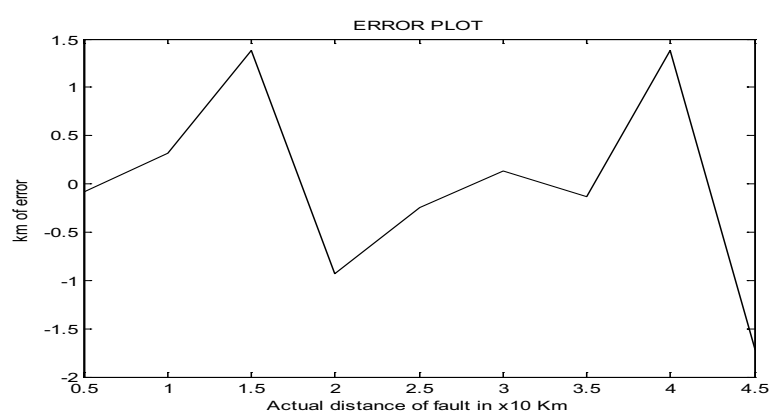

Fig. 5(c)(ii). Fault location error of Hybrid approach for double line to ground fault $(0-50 \mathrm{Km})$

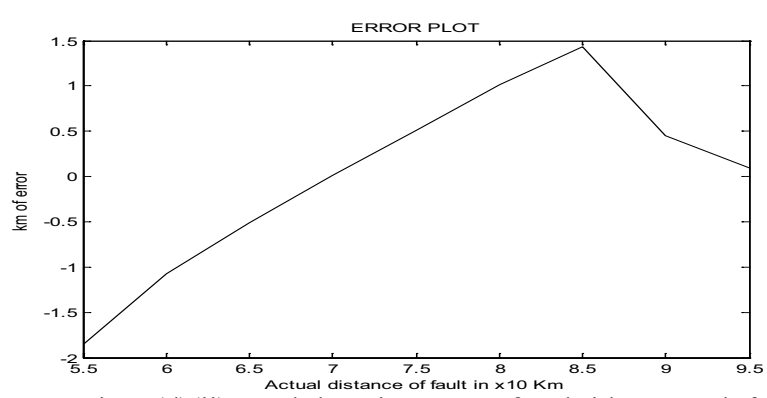

Fig. 5(d)(ii). Fault location error of Hybrid approach for double line to ground fault $(50-100 \mathrm{Km})$

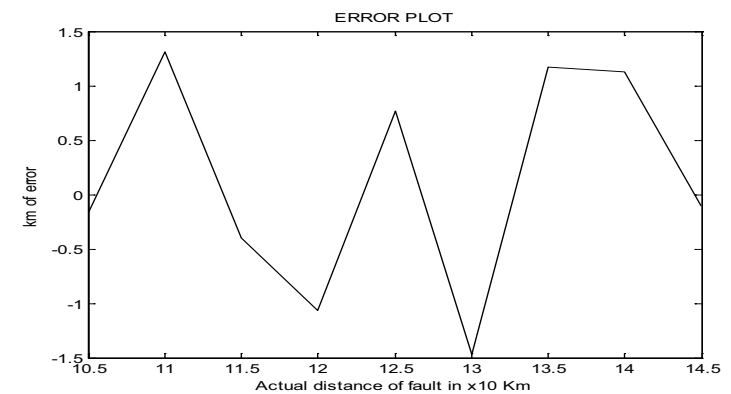

Fig. 5(e)(ii). Fault location error of Hybrid approach for double line to ground fault $(100-150 \mathrm{Km})$

The maximum errors occur from the learning sample and the results of the error at the first stage of location ( $R B F$ based reconstructed input of SVM), magnify the frequency characteristics in the high frequency range and further accurate distance is found by SCALCG based neural network. Fig. 5(c) (ii), Fig. 5(d) (ii) and Fig. 5(e) (ii) are the error plots of (0$50 \mathrm{~km}),(50-100 \mathrm{~km})$ and $(100-150 \mathrm{~km})$ respectively. These are obtained from the testing data that are used to check the proposed solution practically in the present superior approach in all possible type of fault. As seen, the maximum error of FL is limited to $1.852 \mathrm{~km}$ in the worst case and $7.874 \mathrm{e}^{-003} \mathrm{~km}$ in the best case with a short duration of time. The analysis gives the numerical experiments values for the FL given in Table 1, Table 2 and Table 3 respectively, where ' $t$ ' is the target in $\mathrm{km}$, ' $\mathrm{a}$ ' is the actual value in $\mathrm{km}$ and ' $\mathrm{c}$ ' is the error in $\mathrm{km}$. The analysis of the present work proves to be an effective approach in finding the FL in double circuit transmission lines.

The results shown in Fig. 5(c) (ii) to Fig. 5(e) (ii) give the performance of error plot of double circuit for double line to ground fault. Fig. 5(c) (ii) illustrates the error plot using hybrid approach of double circuit for double line to ground fault $(0-50 \mathrm{Km})$ with actual distance of fault (a) on $\mathrm{x}$-axis in $\mathrm{km}$ and error distance of fault (c) on y-axis in $\mathrm{km}$ with $\mathrm{R}_{\mathrm{f}}=40 \Omega, \mathrm{R}_{\mathrm{g}}=10 \Omega$ along with the error value of $0.08555 \mathrm{~km}$ (minimum) and $1.7204 \mathrm{~km}$ (maximum). Similarly Fig. 5(d) (ii) illustrates the error plot using hybrid approach of double circuit for double line to ground fault $(50-100 \mathrm{~km})$ with actual distance of fault (a) on $\mathrm{x}$-axis in $\mathrm{km}$ and error distance of fault (c) on y-axis in $\mathrm{km}$ with $\mathrm{R}_{\mathrm{f}}=40 \Omega, \mathrm{R}_{\mathrm{g}}=$ $10 \Omega$ along with the error value of $7.874 \mathrm{e}^{-003} \mathrm{~km}$ (minimum) and $1.852 \mathrm{~km}$ (maximum). Fig. 5(e) (ii) illustrates the error plot using hybrid approach for double line to ground fault $(100-150 \mathrm{Km})$ with actual distance of fault (a) on $\mathrm{x}$-axis in $\mathrm{km}$ and error distance 
of fault (c) on y-axis in $\mathrm{km}$ with $\mathrm{R}_{\mathrm{f}}=40 \Omega, \mathrm{R}_{\mathrm{g}}=10 \Omega$ along with the error value of $0.10387 \mathrm{~km}$ (minimum) and $1.4698 \mathrm{~km}$ (maximum).

Table 1. Numerical values of the FL error of hybrid approach for double circuit of double line to ground fault $(0-50 \mathrm{~km})$

\begin{tabular}{ccc}
\hline $\begin{array}{c}\text { Actual value in } \\
\text { x 10 km (a) }\end{array}$ & $\begin{array}{c}\text { Target value in } \\
\text { X 10 km (t) }\end{array}$ & $\begin{array}{c}\text { Error value in } \\
\mathrm{km}(\mathrm{c})\end{array}$ \\
\hline 0.58555 & 0.5 & -0.08555 \\
0.68426 & 1.0 & 0.31574 \\
0.11445 & 1.5 & 1.38555 \\
2.9261 & 2.0 & -0.9261 \\
2.7419 & 2.5 & -0.2419 \\
2.8621 & 3 & 0.1379 \\
3.6339 & 3.5 & -0.1339 \\
2.6214 & 4 & 1.3786 \\
6.2204 & 4.5 & -1.7204 \\
\hline
\end{tabular}

Table 2.Numerical values of the FL error of hybrid approach for double circuit of double line to ground fault $(50-100 \mathrm{~km})$

\begin{tabular}{ccc}
\hline $\begin{array}{c}\text { Actual value in } \\
\text { x 10 km (a) }\end{array}$ & $\begin{array}{c}\text { Target value in } \\
\text { X 10 km (t) }\end{array}$ & $\begin{array}{c}\text { Error value in } \\
\mathrm{km} \mathrm{(c)}\end{array}$ \\
\hline 7.352 & 5.5 & 1.852 \\
7.0731 & 6 & 1.0731 \\
7.0103 & 6.5 & -0.5103 \\
6.9921 & 7 & $7.874 \mathrm{e}^{-003}$ \\
6.9875 & 7.5 & 0.51255 \\
6.9857 & 8 & 1.0143 \\
7.0656 & 8.5 & 1.4344 \\
8.5463 & 9 & 0.45369 \\
9.4024 & 9.5 & 0.097563 \\
\hline
\end{tabular}

Table 3. Numerical values of the FL error of hybrid approach for double circuit of double line to ground fault $(100-150 \mathrm{~km})$

\begin{tabular}{ccc}
\hline $\begin{array}{c}\text { Actual value in } \\
\text { x 10 km (a) }\end{array}$ & $\begin{array}{c}\text { Target value in } \\
\text { X 10 km (t) }\end{array}$ & $\begin{array}{c}\text { Error value in } \\
\mathrm{km}(\mathrm{c})\end{array}$ \\
\hline 10.659 & 10.5 & -0.15873 \\
9.6882 & 11 & 1.3118 \\
11.901 & 11.5 & -0.4007 \\
13.069 & 12 & -1.0691 \\
11.735 & 12.5 & 0.76454 \\
14.47 & 13 & -1.4698 \\
12.328 & 13.5 & 1.1723 \\
12.875 & 14 & 1.1246 \\
14.604 & 14.5 & -0.10387 \\
\hline
\end{tabular}

Table 1gives the result of numerical values of the FL error of hybrid approach for double circuit of double line to ground fault $(0-50 \mathrm{~km})$ with target value $(\mathrm{t})$, actual value (a), and error value (c). Table 2 gives the result numerical values of the FL error of hybrid approach for double circuit of double line to ground fault (50-100 km) with target value (t), actual value (a), and error value (c). Table 3 gives the result numerical values of the FL error of hybrid approach for double circuit of double line to ground fault $(100-150 \mathrm{~km})$ with target value ( $\mathrm{t}$ ), actual value (a), and error value (c).

SVM fault location (Robert Salat and Stanislaw Osowski (2004), [10] ) with $\pm 4 \%$ error. The minimum error value of fault location for the proposed method is $7.874 \mathrm{e}^{-003} \mathrm{~km}$ and maximum error value is $1.852 \mathrm{~km}$ representing quite satisfactory results in double circuit fault location using hybrid approach as shown in Fig. 5(c) to Fig. 5(e) and the values are given in Table 1 to Table 3. This application offers increased accuracy under a variety of operating and fault conditions and is not affected by the fault resistance and even provides accurate fault location determination for a time varying fault impedance.

Compare to ANN, SVM is the best method for location of fault because,

(a) BFG based ANN are trained with various set of input pattern and for each set there is a separate set of waveforms where as in SVM analysis only one training pattern is enough for all data.

(b) In ANN locator use the fraction numbers we ourself make them to whole numbers as 0 or 1 . But in SVM there in the method to fit the input analysis for support vectors using reconstruction method, make a boundary region to identify the data for initial location further accurately locate finner adjustment using the $\mathrm{NN}$.

(c) SVM takes less time duration for simulation and also for execution (BFG based ANN $0.0005 \mathrm{Sec}$, SVM $\left.2 \mathrm{e}^{-005} \mathrm{Sec}\right)$

(d) In case of BFG based ANN if there is a fault in the particular line, then that particular line alone be considered for fault location, while in SVM analysis other phases will be considered for fault location, hence SVM analysis is the very effective one to locate the accurate fault location.

(e) Coarse and fine adjustment are appeared, so SVM is more accurate using two stages, hence found the accurate point of the fault location

(f) Finding sampling data is easier, because in SVM are having reduced input data for analysis the system. So no feeding of large set of data for input analysis. Reconstructed inputs are used to implement the system to locate the fault more accurately. 
So it is proven to the compared to be with ANN, SVM NN is better to locate the fault for EHV transmission lines both single and double circuit lines.The analysis of the present work proves to be an effective approach in finding fault location in double circuit EHV transmission lines. The analysis of the present work proves to be an effective approach for finding fault location for double circuit transmission lines.

\section{Conclusion}

In this paper, hybrid approach of the RBF based reconstructed input of SVM and SCALCG based NN method is taken up and proved to be accurate and efficient method for the location of faults. The results are achieved in a very short duration of time $\left(2 \mathrm{e}^{-004} \mathrm{sec}\right)$. The discrimination in the fault location of the proposed method is accurate as it includes the trained voltages and currents as well as frequency which are used. The method comprises the hybrid approach, which involves two steps. In the first step, the RBF based SVM with reconstructed input estimates the initial distance to the phase of fault using the information contained in the positive sequence voltages and currents of faulty phases. Reconstruction approach is involved with reconstructed input values (change the input value to suit the system requirement). The second step improves the final estimation of this distance using SCALCG based NN with the information contained in the high frequency range characteristics. The reconstructed input of SVM makes this approach robust and simple. It is also proposed for more general case nonlinear function as it speeds up things by making the SVM expansion more sparse. The results reached for the location of faults prove the very good performance of this research. SVM fault location (Robert Salat and Stanislaw Osowski (2004), [10] ) with $\pm 4 \%$ error. The maximum errors of fault location for the proposed method is limited to $1.852 \mathrm{~km}$ (double circuit) in the worst case and $7.874 \mathrm{e}^{-}$ ${ }^{003} \mathrm{~km}$ (double circuit) in the best case with a short duration of time in each $50 \mathrm{~km}$ line, the type of system which can locate the fault in EHV double circuit transmission line for double line to ground fault. This method is simple but computationally faster and presents efficient results.

\section{References}

1. A.J. Mazon, J.F .Minambres, M.A. Zorrozua and I. R. Zamora Alvarez-Isasi, New method of fault location on double-circuit two-terminal transmission lines, Electric power system research 35 (1995) 213-219.

2. Q.Y .Xuan, R.K. Aggarwal, A.T. Johns, R.W. Dunn and A. Bennett, A neural network based protection technique for combined $275 \mathrm{kv} / 400 \mathrm{kv}$ double circuit transmission lines, Neurocomputing 23(1998) 59-70.

3. Lin Bo Sheng and S.Elangovan, A fault location algorithm for transmission lines, Electric machines and power systems 26(1998) 991-1005.

4. Lin Bo Sheng and S.Elangovan, A fault location method for parallel transmission lines, Electrical power and energy systems 21(1999) 253-259.

5. A.J. Mazon, I. Zamora, J.F. Minambres, M.A .Zorrozua, J.J.Barandiaran and K.Sagastabeitia, A new approach to fault location in two terminal transmission lines using artificial neural network, Electric power system research 56 (2000) 261-266

6. G.K. Purushothama, A.U. Narendranath, D. Thukaram and K. Parthasarathy, ANN applications in fault locator, Electrical power and energy systems 23(2001) 491-506.

7. S.A. Soliman, M.H. Abdul Rahman, E. Al-Attar and M.E. E1-Hawary, An algorithm for estimating fault location in an unbalanced three phase power system, Electrical power and energy systems 24 (2002) 515-520.

8. Tamer Kawady and Jurgen Stenzel, A practical fault location approach for double circuit transmission lines using single end data, IEEE transactions on power delivery 18 (4) (2003) 1166-1173.

9. M Joorabian, S.M.A. Taleghani Asl and R.K. Aggarwal, Accurate fault locator for EHV transmission lines based on radial basis function neural networks, Electric power system research 71 (2004) 195- 202.

10. Robert Salat and Stanislaw Osowski., Accurate fault location in the power transmission line using support vector machine approach, IEEE transactions on power systems 19 (2) (2004) 979- 986.

11. J. Izykowski, E. Rosolowski and M. Mohan Saha, Locating faults in parallel transmission lines under availability of complete measurements at one end, Modern electric power systems 151(2) (2004) 268-273.

12. L. Sousa Martins, J.F. Martins, V. Fernao Pires and C.M. Alergria, A neural spaces vector fault location for parallel double-circuit distribution lines, Electrical power and energy systems 27 (2005) 225-231.

13. J. Gracia, A.J. Mazon and I. Zamora, Best ANN structures for fault location in single and double-circuit transmission lines, IEEE transactions on power delivery 20 (4) (2005) $2389-2395$.

14. Hosung Jung, Young Park, Moonseob Han, Changmu Lee, Hyunjune Park and Myongchul Shin, Novel technique for fault location estimation on parallel transmission lines using wavelet, Electrical power and energy systems 29(2007) 76-82. 
15. M. Sukumar and Brahma, New fault location method for a single multiterminal transmission line using synchronized phasor measurements, IEEE transactions on power delivery 21(3) (2006) 1148-1153.

16. S.R. Samantaray, P.K. Dash and G. Panda, Distance relaying for transmission line using support vector machine and radial basis function neural network, Electrical power and energy systems 29 (2007) 551-556.

17. Carlos Eduardo de Morais Pereiro and Luiz Cera Zanetta Jr., An optimisation approach for fault location in transmission lines using one terminal data, Electrical power and energy systems 29 (2007) 290-296.

18. Chun Wang, Qing-Quan Jia, Xin-Bin Li and Chun-Xia Dou, Fault location using synchronized sequence measurements, Electrical power and energy systems 29 (2007) 551-556.

19. K. Gayathri and N. Kumarappan, ANN based fault detection and location in EHV transmission line, in Proc. 38-th North American Power Symposium (Carbondale, Illinois US, 2006), pp.559-565.

20. K.Gayathri, N. Kumarappan and C. Devi, An apt method for fault identification and classification on EHV lines using discrete wavelet transform, in Proc. 8-th International Power Engineering Conference (Singapore, 2007), pp. a582-a587.

21. Neculai Andrei, A scaled Nonlinear Conjugate Gradient Algorithm for Unconstrained Optimization, Research in Institute for Informatics, (Center for Advanced Modeling and Optimization, 8-10,Averescu Avenu, Bucharest 1, Romania, E.Mail:nandrei@ici.ro, 2006).

22. K. Gayathri and N. Kumarappan, Accurate fault location on EHV lines using both RBF based support vector machine and SCALCG based neural network, Expert Systems with Applications 37(2010) 8822-8830.
Vitae

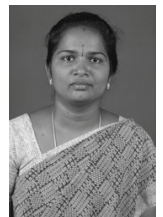

K.Gayathri was born on April 10, 1976. She completed her B.E. degree in Electrical and Electronics in 1999, M.E. degree in Power Systems in 2005 and also awarded her Ph.D in Electrical Engineering in September 2010 at Annamalai University. She is presently a Assistant Professor at Annamalai University, India. She has 20 publications to her credit. Her research interests include power system analysis, fault diagnosis and artificial intelligence techniques to power systems.

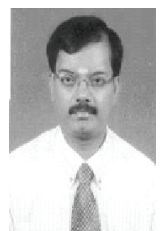

N.Kumarappan was born on November 27, 1961. He was graduated from TCE, Madurai Kamaraj University,1982, post graduated from Annamalai University, 1989 and received his $\mathrm{PhD}$ at CEG (under QIP, AICTE), Anna University, Chennai, 2004. He has 104 publications to his credit. He is presently a Professor in the Annamalai University. His research interests include power system operation and control; power system reliability and artificial intelligence techniques. $\mathrm{He}$ is the Fellow of Institution of Engineer's (India) and Life Member of Indian society of Technical Education. He is the Senior Member of the IEEE (USA). 